\title{
Binaural additivity of loudness in children and adults
}

\author{
BRUCE A. SCHNEIDER \\ University of Toronto, Erindale Campus, Mississauga, Ontario, Canada \\ and \\ ANNABEL J. COHEN \\ University of Prince Edward Island, Charlottetown, Prince Edward Island, Canada
}

\begin{abstract}
Thirty-six different binaural noises were formed by crossing six right-ear intensities of a broadband noise with the same six intensities in the left ear in a $6 \times 6$ factorial design. Children (6-7 years of age) and adults were presented with 2 of these 36 binaural noises on a trial and asked to indicate which noise was louder. In Experiment 1, the left- and right-ear noises were in phase and differed only in intensity. In Experiment 2, the left- and right-ear noises were in opposite phase. For both the children and adults in Experiments 1 and 2, the paired comparison judgments of binaural loudness were shown to satisfy the testable axioms of conjoint measurement (transitivity and double cancellation), permitting the determination of interval scales of loudness for the left ear, right ear, and the sum of the two ears. Power functions provided a good description of the relation between loudness and sound pressure for the left and right ears of both children and adults. For both adults and children, an examination of the pattern of differences in judgments between Experiments 1 and 2 indicated that, when the noises were in phase, the contribution of the right ear to fused loudness was greater than when the noises were presented in counterphase.
\end{abstract}

Although there is an extensive literature on the relationship of loudness to sound intensity (see Marks, 1979b, for a review), almost all of these studies employ adult listeners. Thus, we have little information concerning developmental changes in the perception of loudness. For the most part, this is due to the difficulties in obtaining valid loudness judgments from children.

\section{The Measurement Problem}

In a typical loudness-scaling experiment, subjects are asked either to assign numbers to sounds so that the numbers are proportional to the loudnesses of the sounds or to match number magnitude to loudness. For adult subjects, these numerical judgments, $N$, are typically found to be a power function of sound intensity; that is,

$$
N=k P^{n} \text {, }
$$

where $P$ is sound pressure and $k$ and $n$ are constants. This power-function relationship is often referred to as Stevens's law (Stevens, 1956). To perform this task, however, subjects must be able to understand the ratio properties of numbers. But, as Teghtsoonian (1980) has noted, very few children younger than 8 years are capable of using numbers in this fashion. Thus, the use of numerical estima-

\footnotetext{
This research was supported by a grant from the Medical Research Council of Canada. The authors extend their thanks to Sandra Piasecki and Jane Carey for conducting the experiments. Correspondence should be directed to B. A. Schneider, Department of Psychology, University of Toronto, Erindale Campus, 3359 Mississauga Rd., Mississauga, ON, Canada L5L IC6 (e-mail: schneid@psych.utoronto.ca).
}

tion techniques is unlikely to give valid results in young children.

Bond and Stevens (1969) and Teghtsoonian (1980) have suggested that a better strategy for evaluating loudness in children would be to have them perform a cross-modality match. Bond and Stevens trained 5-year-old children to adjust the brightness of a light to match the loudness of a sound. Teghtsoonian had children in one condition adjust the length of a line to match the loudness of a sound and in another condition to adjust the loudness of a sound to match the length of a line (a balanced cross-modality matching procedure). Although both Bond and Stevens (1969) and Teghtsoonian (1980) were able to obtain consistent loudness matches in this fashion for children as young as 4 years of age, the relationship of such matches to perceived loudness is uncertain. The outcome of a match of loudness to line length will depend not only on the relation between loudness and sound intensity, but also on the relation between perceived line length and physical length. Because both of these relationships are unknown in children, we cannot use the outcome of a cross-modal match to uncover the form of the loudness function. To see that this is true, we need only note that both Stevens's power law and Fechner's logarithmic law $[L=a \log (P)]$ predict that cross-modality matches should be linearly related on $\log -\log$ coordinates. Thus, cross-modal matches, by themselves, do not permit the determination of a loudness function in children.

Collins and Gescheider (1989) attempted to measure both loudness and line length using the absolute magnitude estimation (AME) paradigm before having the adults 
and children (4-7 years of age) in that experiment perform a cross-modality match between line length and loudness. In absolute magnitude estimation, the subject is encouraged to use her or his own scale and to not compare one stimulus to another when assigning a number. They found reasonable power functions for the growth of loudness in children provided that they corrected for certain problems in children's AMEs. Two problems were noted. First, some children did not use numbers smaller than 1.0. At the other extreme, some children used large numbers that they could not consistently order. In determining least squares estimates of the exponent, Collins and Gescheider used only the largest stimulus that was assigned the number 1 in their least squares estimates of the exponent and eliminated all numerical responses that fell above the numerical limit beyond which children could not consistently order numbers. They noted that once children's judgments were corrected for these two factors, they produced power functions with exponents similar to that of adults.

Note, however, that in the Collins and Gescheider (1989) study, young children experienced some difficulty in using numbers appropriately. First of all, a comparison of the adult and child magnitude estimation function (see their Figure 1) shows that children do not use numbers below 1 . Second, an examination of their Table I shows that the upper numerical limit beyond which they cannot order numbers appropriately ranges from 20 to 100 . Thus, children, at best, have only a restricted numerical scale that they can match to line lengths. We need, therefore, a method of measuring loudness in children that can determine the precise form of the loudness function, but whose requirements are well within the cognitive capabilities of very young children. We will show below that conjoint measurement techniques, applied to binaural loudness judgments, satisfy these criteria.

\section{Binaural Loudness}

When identical sounds, differing only in amplitude, are presented to the left and right ears, the listener perceives a single fused sound whose perceived location and loudness is a function of both the left- and right-ear intensities. Let $L\left(P_{a}, P_{p}\right)$ represent the loudness of a binaurally fused sound where the subscripts $a, b, c$, and so forth, specify the sound pressures of the left-ear sounds and the subscripts $p, q, r$, and so forth, specify the intensities of the right-ear sounds. A simple model for the loudness of this fused sound is

$$
L\left(P_{a}, P_{r}\right)=L_{\mathrm{L}}\left(P_{a}\right)+L_{\mathrm{R}}\left(P_{r}\right),
$$

where $L_{\mathrm{L}}$ and $L_{\mathrm{R}}$ are left- and right-ear loudnesses. In words, Equation 2 says that the loudness of the fused sound is the sum of the left- and right-ear loudnesses.

Several studies (Algom \& Marks, 1984; Levelt, Riemersma, \& Bunt, 1972; Marks, 1978, 1979a, 1980) have shown that the judgments of subjects are, for the most part, consistent with this additive model (but see Gigerenzer \& Strube, 1983). These studies also show that both left- and right-ear loudness is a power function of sound intensity; that is,

$$
L_{\mathrm{L}}(P)=k_{\mathrm{L}} P^{n_{\mathrm{L}}} \text { and } L_{\mathrm{R}}(P)=k_{\mathrm{R}} P^{n_{\mathrm{R}}}
$$

where $k_{\mathrm{L}}, k_{\mathrm{R}}, n_{\mathrm{L}}$, and $n_{\mathrm{R}}$ are constants. Finally, most of these studies find that $n_{\mathrm{L}}$ and $n_{\mathrm{R}}$ are roughly equal, suggesting left and right symmetry for binaural additivity.

\section{The Measurement Technique}

Most of the studies of the relation between binaural loudness and sound intensity have obtained numerical judgments of the loudnesses of fused sounds from adult subjects. But as we have already seen, the use of numerical estimation techniques with children is, at best, risky. Luce and Tukey (1964), however, have provided a technique, called conjoint measurement, by which loudness scales can be derived from judgments of which of two binaurally fused sounds is louder. Clearly, such comparative loudness judgments should be well within the capabilities of even very young children (Jensen \& Neff, 1993; Maxon \& Hochberg, 1982). For this reason, we have employed these conjoint measurement techniques to study binaural loudness in children.

In each of two experiments, independent groups of both children and adults were asked to compare two binaurally fused sounds and indicate which of the two sounds was louder (the first or the second). A set of 36 binaurally fused sounds was created by crossing 6 left-ear intensities of a broadband noise with the same 6 intensities presented to the right ear. On a trial, the subject was presented with two fused sounds from this set in a two-interval forced-choice (2IFC) task and asked to indicate which sound was louder. Thus, the subject might be asked to indicate, for example, whether $(50,74)$ was louder than $(56,68)$ or vice versa, where the numbers in the parentheses specify the left and right ear intensities in decibels SPL. If the additive model (Equation 2) holds, then

$$
\begin{aligned}
(50,74) & \geq(56,68) \\
\text { iff } L_{\mathrm{L}}(50)+L_{\mathrm{R}}(74) & \geq L_{\mathrm{L}}(56)+L_{\mathrm{R}}(68) .
\end{aligned}
$$

Note that for certain of the pairs, we know exactly what the outcome of the judgment should be. For example, if asked to compare $(50,74)$ with $(62,80)$, a subject who is following instructions should judge the latter as louder than the former, because the left and right intensities in the second sound are both greater than they are in the first sound. During a session, subjects were occasionally presented with such pairs and visually reinforced for making the appropriate response. Performance on such pairs was monitored to ensure that subjects were following instructions.

\section{Scale Construction and Validation}

Luce and Tukey (1964) have shown that loudness comparisons must satisfy certain constraints if left- and rightear loudnesses are to be considered as additive. First of all, the comparisons must be transitive; that is, 


$$
\begin{aligned}
\left(P_{a}, P_{p}\right) & \geq\left(P_{b}, P_{r}\right) \\
\text { and }\left(P_{b}, P_{r}\right) & \geq\left(P_{c}, P_{s}\right) \Rightarrow\left(P_{a}, P_{p}\right) \geq\left(P_{c}, P_{s}\right) .
\end{aligned}
$$

A second constraint, and one that is not so immediately obvious, is referred to as double cancellation. If loudnesses are additive, then

$$
\begin{aligned}
\left(P_{a}, P_{p}\right) & \geq\left(P_{b}, P_{r}\right) \\
\text { and }\left(P_{b}, P_{s}\right) & \geq\left(P_{c}, P_{p}\right) \Rightarrow\left(P_{a}, P_{s}\right) \geq\left(P_{c}, P_{r}\right) .
\end{aligned}
$$

We can identify the reason for this condition if we substitute, in each of the pairs in Equation 6, their corresponding loudnesses as determined by Equation 2. For the inequalities on the left of the implication sign, we have

$$
L_{\mathrm{L}}\left(P_{a}\right)+L_{\mathrm{R}}\left(P_{p}\right) \geq L_{\mathrm{L}}\left(P_{b}\right)+L_{\mathrm{R}}\left(P_{r}\right)
$$

and

$$
L_{\mathrm{L}}\left(P_{b}\right)+L_{\mathrm{R}}\left(P_{s}\right) \geq L_{\mathrm{L}}\left(P_{c}\right)+L_{\mathrm{R}}\left(P_{p}\right) .
$$

Adding these two inequalities together yields

$$
\begin{aligned}
L_{\mathrm{L}}\left(P_{a}\right) & +L_{\mathrm{R}}\left(P_{p}\right)+L_{\mathrm{L}}\left(P_{b}\right)+L_{\mathrm{R}}\left(P_{s}\right) \\
& \geq L_{\mathrm{L}}\left(P_{b}\right)+L_{\mathrm{R}}\left(P_{r}\right)+L_{\mathrm{L}}\left(P_{c}\right)+L_{\mathrm{R}}\left(P_{p}\right) .
\end{aligned}
$$

Eliminating common terms on both sides of the inequality leaves

$$
L_{\mathrm{L}}\left(P_{a}\right)+L_{\mathrm{R}}\left(P_{s}\right) \geq L_{\mathrm{L}}\left(P_{c}\right)+L_{\mathrm{R}}\left(P_{r}\right) .
$$

Thus, if loudness is additive, double cancellation must hold. Krantz, Luce, Suppes, and Tversky (1971) have shown that if, for all effective purposes, double cancellation is not violated, the system is additive. We can, therefore, test whether the loudness comparisons are consistent with an assumption of an additive model (Equation 2). This test, combined with the test pairs for which the direction of the loudness comparison is known, a priori, can be used to evaluate the extent to which the children are actually judging loudness.

\section{Deriving Loudness Scales}

Provided that the system is additive, we can determine scale values for the loudnesses of the left-ear sounds, the loudnesses of the right-ear sounds, and the loudnesses of the fused sounds, which are, for all practical purposes, unique up to addition and multiplication by a constant. Specifically, if $S_{\mathrm{L}}$ and $S_{\mathrm{R}}$ are the loudness-scale values derived from the paired comparison judgments for left and right signals, respectively, then $S_{\mathrm{L}}$ and $S_{\mathrm{R}}$ are related to the "true" loudness values, $L_{\mathrm{L}}$ and $L_{\mathrm{R}}$, by the following transformations

$$
S_{\mathrm{L}}=\alpha L_{\mathrm{L}}+\beta_{\mathrm{L}}, S_{\mathrm{R}}=\alpha L_{\mathrm{R}}+\beta_{\mathrm{R}},
$$

where $\alpha, \beta_{\mathrm{L}}$, and $\beta_{\mathrm{R}}$ are constants. In other words we can achieve interval scale measurement of loudness in a binaural summation paradigm.

\section{Binaural Additivity and Interaural Phase}

When the left- and right-ear sounds are in phase and of equal intensity, adult subjects will tend to perceive the sound as centered in their heads. If, however, the left-ear intensity is greater than the right-ear intensity, they will perceive the sound as displaced toward the left ear, with the extent of the displacement varying with the interaural intensity difference. This means that in the conjoint loudness paradigm, the two sounds to be compared might differ not only in loudness but also with respect to lateral position. Levelt et al. (1972) have shown that adult subjects can make consistent loudness comparisons independent of differences in lateralization. However, the possibility that the judgments of children might be influenced by perceived lateral position remained. In an attempt to control for such a factor, we had adults and children not only compare the loudnesses of in-phase noises (Experiment 1) but also the loudnesses of counterphase noises (Experiment 2).

When left- and right-ear noises are in counterphase, their interaural differences are not consistent with those expected of a single source. Consequently, the resulting noise tends to be perceived as more diffuse and less lateralized (see Durlach \& Colburn, 1978, p. 375, for a discussion of these issues). To investigate whether loudness summation in children was influenced by the degree of fusion and lateralization, children and adults were tested with both in-phase and counterphase noise.

\section{METHOD}

\section{Subjects}

Twenty children were recruited from local schools, parent groups, and letters sent to nearby families for Experiment $1 ; 25$ children were similarly recruited for Experiment 2 . The 40 adult participants ( 20 in each experiment) were staff and students associated with the Department of Psychology at the University of Toronto. The children were between 6.08 and 7.33 years old (mean age $=6.91$ years). The adults were between 19 and 27 years of age (mean age $=22.25$ years). No subjects had any known auditory pathology, and all were free of colds on test days.

\section{Apparatus}

The signal was broadband noise. It was obtained by passing the output of a General Radio (Model 1381) noise generator through a Wavetek (System 716) filter. The spectrum level of the noise, after filtering, was flat up to $5 \mathrm{kHz}$ and declined at a rate of $115 \mathrm{~dB} /$ octave thereafter. This signal was then split into two channels, each channel consisting of an electronic switch (rise/decay time of $10 \mathrm{msec}$ ) and a programmable attenuator. The electronic switches controlled signal presentations, and the programmable attenuators controlled signal intensities. Each channel drove one side of a matched pair of earphones (TDH-49). In Experiment 1, the interaural phase was $0^{n}$; in Experiment 2, an interaural phase shift of $180^{\circ}$ was obtained by reversing the polarity of the input to one of the earphones.

\section{Procedure}

Trial structure. A trial consisted of the sequential presentation of two binaural sounds (duration $=744 \mathrm{msec}$ ) separated by an interstimulus interval of $744 \mathrm{msec}$. Each trial began $50 \mathrm{msec}$ after an initiating buttonpress was made by an experimenter, who was also present in the booth, when she judged the child to be in an attentive state. The child was then asked to press one of two buttons to indicate which of the two sounds was louder. After initial training, the children almost never had to be prompted to respond after the presentation of the two sounds. No reinforcement was given on test trials. On control trials, correct responses were reinforced by illumi- 
nating and activating one of four mechanical toys located in a box to the left of the child.

The testing situation was identical for the adults, except that the experimenter was not in the chamber during testing, subjects pressed the button initiating the trial, and only the light and not the toy was presented for correct responses on training and control triais. No adult subject participated in more than two sessions on a single day.

The 36 fused sounds that constituted the stimulus set were created by crossing six intensity values $(50,56,62,68,74$, and $80 \mathrm{~dB})$ of the noise in the left ear with the same six values of the noise in the right ear. With 36 fused sounds, there are $(36 \times 35) / 2=630$ possible paired comparisons of distinct sounds. However, in 405 of these 630 comparisons, the left- and right-ear intensities in one pair were greater than or equal to the left- and right-ear intensities in the other pair. If monaural loudness is monotonic with intensity, then the pair with the greater intensity in both ears should be judged as louder; that is,

$$
P_{a} \geq P_{b} \text { and } P_{p} \geq P_{q} \Rightarrow\left(P_{a}, P_{p}\right) \geq\left(P_{b}, P_{q}\right),
$$

or

$$
P_{a} \leq P_{b} \text { and } P_{p} \leq P_{q} \Rightarrow\left(P_{a}, P_{p}\right) \leq\left(P_{b}, P_{q}\right) .
$$

Consequently, paired comparisons of this type were not presented on test trials. Thus, on test trials, 1 of the remaining 225 comparisons for which the direction of the loudness judgment could not be specified a priori was presented.

On control trials, one of the following five pairs was randomly selected: $(50,50)$ versus $(56,56) ;(56,56)$ versus $(62,62) ;(62,62)$ versus $(68,68) ;(68,68)$ versus $(74,74)$; and $(74,74)$ versus $(80,80)$. Note that, for each of the pairs, there is a $6-\mathrm{dB}$ separation in leftand right-ear intensities, and that the direction of the loudness judgment is predetermined and could be reinforced. Each child participated in six test sessions on different days. During the first session, only control pairs were presented. Each of the remaining five test sessions consisted of 45 test trials and 10 control trials. The 225 test pairs were distributed randomly over the five sessions. Trials 1 , $7,13,19,25,31,37,43,49$, and 55 were control trials. On control trials, each of the five control comparisons was randomly presented twice during the session. The order of presentation of the stimuli within a trial was randomly determined. Thus, each subject was exposed once to one of the 225 test comparisons and asked to indicate which of the two fused sounds (the first or the second) was louder.

\section{RESULTS}

\section{Control Pairs}

In control pairs, the right- and left-ear intensities of one of the pairs was always $6 \mathrm{~dB}$ greater than the right- and left-ear intensities in the other pair. Therefore, if the children were actually judging loudness, we would expect them to pick the sound whose left- and right-ear intensities were greater. The average percentage correct on these control trials in Experiment 1 was $91.6 \%$ for the children and $98.4 \%$ for the adults. In Experiment 2, the averages were $93.4 \%$ and $98.1 \%$ for the children and adults, respectively. Although children performed less well than adults, the scores on the control pairs are sufficiently high to support the contention that the children were actually judging the loudnesses of the fused sounds.

\section{Loudness Comparisons and Lateralization}

On noncontrol comparisons, if the left-ear intensity of the first sound was greater than the left-ear intensity of the second sound, the right-ear intensity of the second sound was always greater than the right-ear intensity of the first sound. Conversely, if the right-ear intensity of the first sound was greater than the right-ear intensity of the second sound, the left-ear intensity of the second sound was always greater than the left-ear intensity of the first sound. Therefore the two sounds should always be lateralized to opposite sides of the midline. If children were to experience a greater degree of difficulty than adults in comparing sounds differing in lateral position, then we might expect their judgments to be more variable than those of adults. To check for this, we examined all 70 paired comparisons in which either the left- or right-ear intensity in one of the fused noises was at least $12 \mathrm{~dB}$ greater than either the left- or right-ear intensity of the other fused noise [e.g., $(56,80)$ vs. $(68,56)$ or $(74,50)$ vs. $(56,62)]$. Under these conditions, we would expect the sound whose leftor right-ear intensity was at least $12 \mathrm{~dB}$ greater than either the left- or right-ear intensities of its comparison to be judged as louder. For the in-phase noise condition, the adults judged the sound whose left- or right-ear intensity was at least $12 \mathrm{~dB}$ greater than the left- or right-ear intensities of the comparison sound to be louder on $96.6 \%$ of the trials; the children judged them to be louder on $90.4 \%$ of the trials. Although adults had a slightly higher percentage than children, both groups were capable of making consistent loudness judgments when the two sounds to be compared were lateralized to different sides of the head and the intensity difference between the two sounds was sufficiently large in order for one to be confident as to the expected direction of the comparison.

In Experiment 2, where the sounds were in counterphase, $96.7 \%$ of the adults and $90.0 \%$ of the children judged the sound with the greater $(\geq 12 \mathrm{~dB})$ left- or rightear intensity as louder. Thus, whether or not the sounds were in phase (and, presumably, well lateralized) or in counterphase (and, presumably, more diffuse and less well lateralized) had no discernible effect on the percentage of times the sound with the more intense left- or right-ear intensity was judged as louder in all 70 of these loudness comparisons for both children and adults. Moreover, for these comparisons, the only difference between children and adults was that the percentages were slightly higher for adults than for children. Given that percentages were also slightly higher for adults for the control stimuli, it seems reasonable to conclude that although children might be slightly more variable than adults, both groups are capable of making consistent loudness judgments that are independent of the laterality of the sounds being compared.

\section{Transitivity and Double Cancellation}

The data for each subject consisted of her or his judgment of which of the two fused sounds was louder for each of the 225 comparisons. If $\left(P_{a}, P_{p}\right)$ was judged to be louder than $\left(P_{b}, P_{q}\right)$ by more than one-half of the subjects, we write $\left(P_{a}, P_{p}\right)>\left(P_{b}, P_{q}\right)$. For the adult subjects in Experiment 1 , there were four pairs such that exactly one- 
half of the subjects judged one of the two fused sounds to be louder than the other. In that case, we write $\left(P_{a}, P_{p}\right)=$ $\left(P_{b}, P_{q}\right)$. For the children in Experiment 1 , there were six pairs that were judged equal. In Experiment 2, there were four pairs judged equal by the adults. In all of the pairs judged equal by children and adults in both experiments, there was no more than a 6 -dB difference between the higher intensity components of the two sounds. There were, of course, no equal pairs for the children in this Experiment 2 because an odd number of children were tested. However, in all of the cases where the direction of the comparison was within I vote of a tie, there was also no more than a 6-dB difference between the higher intensity components of the two sounds. Thus, for both adults and children, close decisions were limited to pairs with an intensity separation of no more than $6 \mathrm{~dB}$ between the more intense ear component of the two sounds.

To check for transitivity in each experiment, we attempted to order the fused sounds such that whenever subjects judged $\left(P_{a}, P_{p}\right) \geq\left(P_{b}, P_{q}\right)$, the fused sound $\left(P_{a}, P_{p}\right)$ was assigned a higher rank than $\left(P_{b}, P_{q}\right)$; that is,

$$
\text { If }\left(P_{a}, P_{p}\right) \geq\left(P_{b}, P_{q}\right) \text {, then } N\left(P_{a}, P_{p}\right)>N\left(P_{b}, P_{q}\right) \text {, }
$$

where $N$ stands for the ordinal position of the pair. If there are no violations of transitivity, an ordering of the fused sounds can be found which does not violate Condition 12 . If, on the other hand, there are violations of transitivity, there is no ordering of the fused sounds that will not lead to a violation of Condition 12. For example, if $\left(P_{a}, P_{p}\right)>$ $\left(P_{b}, P_{q}\right)$ and $\left(P_{b}, P_{q}\right)>\left(P_{c}, P_{r}\right)$ but $\left(P_{c}, P_{r}\right)>\left(P_{a}, P_{p}\right)$, the three pairs in question cannot be ordered without a violation of Condition 12. An ordering was sought that resulted in the minimal number of violations of Condition 12. For the adults in Experiments 1 and 2, the number of violations of transitivity were 0 and 1 , respectively. For children, the number of violations was 1 in Experiment 1 and 3 in Experiment 2. Given the relatively low number of violations, we conclude that the transitivity requirement was satisfied for both children and adults.

To test for violations of double cancellation, all possible cases in which the antecedent conditions (see Equation 6) were met were examined. For the adults in Experiment 1 , there were 210 cases in which the antecedent condition held and 3 violations of double cancellation, for a failure rate of $1.43 \%$. For the adults in Experiment 2, there were 196 cases in which the antecedent conditions held and 3 violations of double cancellation, for a failure rate of $1.5 \%$. For the children in Experiment 1, there were 185 cases in which the antecedent conditions held and a $2.16 \%$ failure rate. In Experiment 2, there were 199 tests of double cancellations and a failure rate of $3.0 \%$ These failure rates are comparable to those observed for both individual and group data in paired comparison experiments on loudness additivity (Levelt et al., 1972; Schneider, 1988). These results suggest that judgments of loudness satisfy to a reasonable degree both transitivity and double cancellation. Therefore, we conclude that binaural loudness is additive for both children and adults for in-phase and counterphase noise conditions.

\section{Loudness Scale Values}

The 225 comparisons of fused tones for both children and adults were used as an input to a conjoint scaling program (Bissett \& Schneider, 1991) designed to assign values, $S$, to each of the stimuli such that whenever

$\left(P_{a}, P_{p}\right) \geq\left(P_{b}, P_{q}\right)$, then $S\left(P_{a}\right)+S\left(P_{p}\right) \geq S\left(P_{b}\right)+S\left(P_{q}\right)$.

Of course, in errorful data, it is not possible to do this perfectly; hence, the program minimizes $G$, where $G$ is the ratio of the number of violations of Condition 13 to the total number of determinant comparisons in the experiment (the number of comparisons minus the number of tied comparisons). If $G=0$, then an additive combination of values, $S$, assigned to left- and right-ear loudnesses perfectly predicts subjects' judgments of which of the two fused sounds is judged louder. For the adults, the values of $G$ were .009 and .014 for Experiments 1 and 2 , respectively. For children, the values were .014 and .031 for Experiments 1 and 2, respectively.

These values of $G$ can be used to estimate the degree to which the values, $S$, obtained from this program actually represent interval scale measurement. To accomplish this, the index of coordinate metric recovery $(C M)$ was estimated. $C M$ is the squared Pearson correlation coefficient between the true loudnesses of the left- and right-ear intensities (which presumably determined the direction of the 225 loudness comparisons using Equation 2) and the values produced by the program. Hence, $C M$ varies between 0 and 1 , and $C M=1$ means that the true loudnesses have been perfectly recovered. In no empirical investigation using these techniques are the true values known, but Bissett and Schneider (1991) show how $C M$ can be estimated given the number of stimuli and the value of $G$. Hence, if the estimated value of $C M$ is sufficiently high, the values obtained from the conjoint program can properly be regarded as interval-scale measurement. $C M$ was estimated to be .999 or above in all cases. Given the high estimated value of $C M$, the projection values for both left- and right-ear intensities can be considered as interval scales of loudness.

Figure 1 plots the projection values as a function of decibels of sound pressure for the left and right ears of both children and adults in Experiments 1 and 2. If loudness is a power function of sound pressure, the projection values should be linearly related to sound pressure raised to a power; that is,

$$
\begin{aligned}
& S_{\mathrm{L}}=\alpha \cdot k_{\mathrm{L}} \cdot P^{n_{\mathrm{L}}}+\beta_{\mathrm{L}}, \\
& S_{\mathrm{R}}=\alpha \cdot k_{\mathrm{R}} \cdot P^{n_{\mathrm{R}}}+\beta_{\mathrm{R}} .
\end{aligned}
$$

An iterative least squares procedure was used to find the best fitting values of $\alpha k_{\mathrm{L}}, \alpha k_{\mathrm{R}}, n_{\mathrm{L}}, n_{\mathrm{R}}, \beta_{\mathrm{L}}$, and $\beta_{\mathrm{R}}$. The curves in Figure 1 represent the best fitting power functions to the data. Figure 1 shows that power functions provide a good description of the relation between loudness and sound pressure for the left and right ears of both children and adults in Experiments 1 and 2. Note that for the children in Experiment 1 (in-phase noise), the leftand right-ear loudness functions are indistinguishable. For 


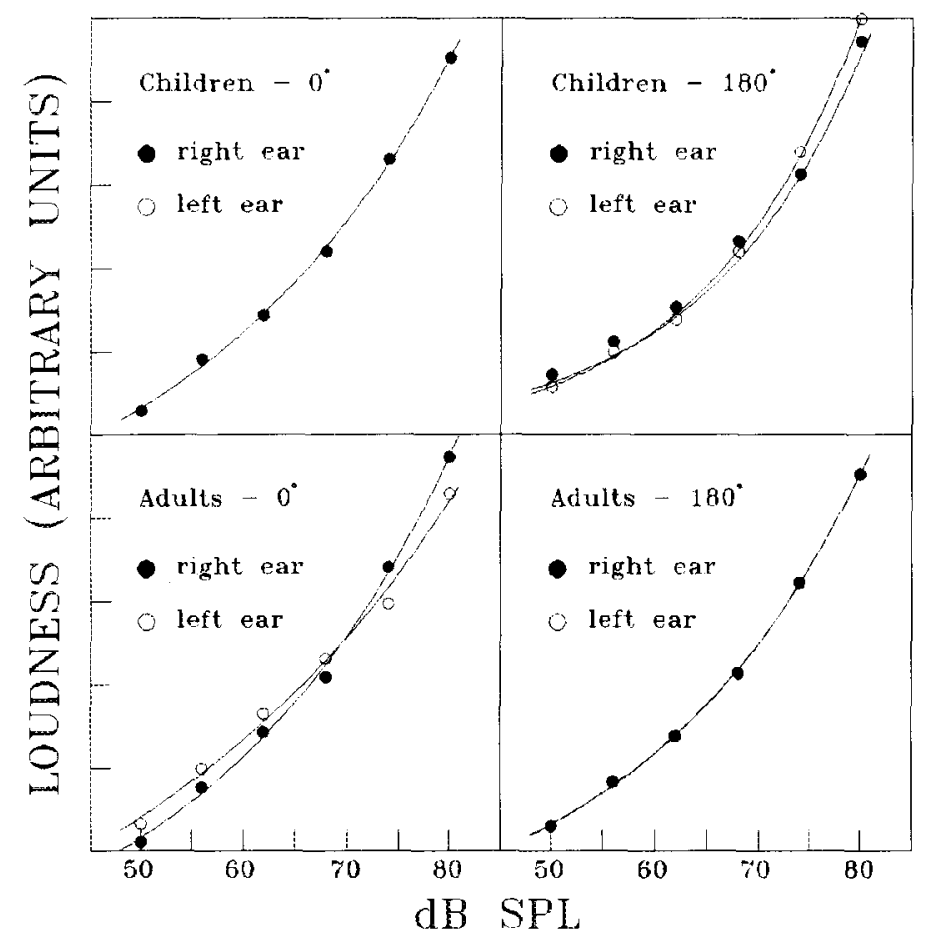

Figure 1. Loudness projections (arbitrary units) as a function of decibels of sound pressure for the left and right ears of adults and children for in-phase and counterphase noise.

the adults in this experiment, however, the right-ear loudness function is slightly steeper and has a more extensive range than the left-ear loudness function, suggesting that the right ear makes a greater contribution to binaural loudness than the left ear. The exponents of the power functions fit to the childrens' data were .34 and .34 for the left and right ears; for the adults, the exponents were .55 and .57 for the left and right ears, respectively.

The pattern of results in Experiment 2 is different. Here the left- and right-ear loudness functions for the adults are essentially identical, while the left-ear loudness function for children has both a larger range and a steeper growth function than the right-ear loudness function. Thus, when noises are presented in counterphase, the left ear of children appears to contribute more to binaural loudness than does the right ear. The exponents of the power functions fit to the left and right ears of the children were .35 and .25 , respectively. For the adults, the left- and rightear exponents were .38 and .38 .

\section{In-Phase Versus Counterphase Noise}

A comparison of in-phase and counterphase conditions suggests that the contribution of the right ear to binaural loudness is greater for in-phase than for counterphase presentations in both children and adults. In adults, the left and right ears contribute about equally to overall loudness in the counterphase conditions, with the contribution of the left ear being somewhat less than that of the right ear in the in-phase condition. Thus, the right ear is given greater weight in the in-phase than in the counterphase condition. In children, the right ear contributes less to binaural loudness in the counterphase condition than does the left ear, while in the in-phase condition its contribution is equal to that of the left ear. Thus, for children, too, the right ear is given greater weight when the noise is in phase than when the noise is counterphase.

To test whether or not this observed pattern was statistically significant, the following analysis was performed. Recall that the paired comparisons tested in this experiment were all of the form that if the right-ear intensity in pair 1 was greater than the right-ear intensity in pair 2 , then the left-ear intensity in pair 2 was greater than the left-ear intensity in pair 1 . Now consider the adult subjects in Experiments 1 and 2. Any differences in the loudness functions between Experiments 1 and 2 reflect the fact that the judgments of some of the 225 comparisons made by the subjects in Experiment 1 were different from those made by the subjects in Experiment 2. In fact, of the 225 comparisons, the subjects in Experiments 1 and 2 agreed on 208 of them. On 17 comparisons, however, when the subjects in Experiment 1 judged pair 1 as louder than pair 2, the subjects in Experiment 2 judged that pair 2 was louder than pair 1 . If these 17 disagreements reflected only random error, we would expect that on the 17 pairs in which the subjects disagreed, the subjects in Experiment 1 would choose the pair whose right-ear intensity was greater about half the time. However, for these 17 comparisons, the Experiment 1 subjects always chose the sound 
whose right-ear intensity was greater. By a binomial test, this is a highly significant difference $(p<.000025$, twotailed). A similar pattern held for the children. Of the 25 comparisons on which the children in the two groups disagreed, the Experiment 1 children chose the pair with the more intense right-ear sound exactly 24 out of 25 times ( $p<.000005$, two-tailed).

Recall that these are between-group comparisons. To obtain a within-group comparison, we attempted to recall as many of the Experiment 1 adults that we could so that we could test them with counterphase noises. Nineteen of the original 20 subjects agreed to repeat the experiment with counterphase noises. These 19 subjects disagreed about which of two pairs was louder in 32 of the comparisons. In 31 of these cases, when the noises were in phase, they chose the pair with the more intense rightear sound ( $p<.000001$, two-tailed). Clearly, the right ear is weighted more heavily when noises are in phase than when they are in counterphase.

It should also be noted that, on average, the loudness functions were somewhat steeper for in-phase than for counterphase noise in both children and adults. In adults, the average of the left- and right-ear exponents for inphase noise was .56; the average of the left- and right-ear exponents for counterphase noise was .38. For children, the average of the left- and right-ear exponents for in-phase noise was .34; for counterphase noise, the average was .30. Although the pattern of values for the exponents indicate that power-function exponents are larger for inphase than for counterphase noise for both adults and children (and, in general, larger for adults than for children in all conditions), it is difficult to tell, for two reasons, whether or not these differences are significant. First, because we employed only a $30-\mathrm{dB}$ range, the change in form of the loudness function when the exponent changes, say, from .55 to .38 , is not large. This can be seen by comparing the fitted functions to the left ear of the adults in the in-phase and counterphase conditions in Figure 1, where the corresponding exponents were .55 and .38 , respectively. Second, because there is no error theory for the loudness projection values, we cannot test whether this exponent difference is significant. Therefore, we cannot evaluate whether or not these exponent differences are statistically significant.

\section{DISCUSSION}

\section{Additivity and Scale Validity}

The low rates of failure for transitivity and double cancellation in the adult data support an additive model for binaural loudness of broadband noise. In this respect, the present study is consistent with the findings of Algom and Marks (1984), Levelt et al. (1972), and Marks (1978, 1979a, 1980), which support an additive model for the binaural summation of pure tones. Marks (1980), however, has reported some experimental results that suggest that binaural additivity does not hold for broadband noise.
In his experiment, subjects gave magnitude estimates of the binaural loudness of a broadband noise. As in the present experiment, different fused noises were produced by crossing a set of left- and right-ear intensities in a factorial design. An analysis of variance of the matrix of loudness estimates resulting from the crossing of left- and right-ear intensities produced a significant interaction term indicating a statistically significant deviation from additivity. However, as Anderson $(1970,1974)$ and Carterette and Anderson (1979) have pointed out, deviations from additivity in such a matrix may simply be due to nonlinear response biases in the magnitude estimates. Suppose, for instance, that loudness was additive (Equation 2) but that magnitude estimates were nonlinearly related to loudness. In that case, we would have $\mathrm{ME}=f\left[L\left(P_{a}, P_{p}\right)\right]=$ $f\left[L_{\mathrm{L}}\left(P_{a}\right)+L_{\mathrm{R}}\left(P_{p}\right)\right]$, where ME is the magnitude estimate of the loudness of a fused sound and $f$ is a nonlinear but monotonic function relating magnitude estimate to fused loudness. Clearly, the transformed data, MEs, need not be additive even though the underlying loudness representation is additive. Because numerous experiments involving judgments of sensory sums, sensory differences, and sensory ratios have demonstrated the existence of nonlinear response biases in numerical estimates (Algom \& Marks, 1984; Anderson, 1974; Curtis, Attneave, \& Harrington, 1968; Curtis \& Rule, 1972; Fagot, Steward, \& Kleinknecht, 1975; Marks, 1978; Rule, Curtis, \& Markley, 1970; Rule, Laye, \& Curtis, 1974; Schneider, Parker, Valenti, Farrell, \& Kanow, 1978), testing for additivity using direct estimates of loudness may not be a valid test for additivity of loudness. Loudness might indeed be additive while our measures are not.

A more serious problem for the binaural additivity of noise arises when loudness matches are obtained between monaural noises and binaural noises (Irwin, 1965; Marks, 1980; Scharf, 1968; Scharf \& Fishken, 1970). Let $L_{\mathrm{m}}(P)$ be the function that relates monaural loudness to sound pressure. Assuming identical loudness functions in both ears and binaural additivity, binaural loudness should be twice the monaural loudness, that is, $L_{\mathrm{b}}(P)=$ $2 L_{\mathrm{m}}(P)$. Consider a loudness matching experiment in which the sound pressure of a monaural noise, $P_{\mathrm{x}}$, is found such that its loudness matches the loudness produced by a binaural noise, $P_{\mathrm{s}}$. This match should occur when $L_{\mathrm{m}}\left(P_{\mathrm{x}}\right)=$ $L_{\mathrm{b}}\left(P_{\mathrm{s}}\right)=2 L_{\mathrm{m}}\left(P_{\mathrm{s}}\right)$. If we solve these equations for $P_{\mathrm{x}}$, we have $P_{\mathrm{x}}=L_{\mathrm{m}}^{-1}\left[2 L_{\mathrm{m}}\left(P_{\mathrm{s}}\right)\right]$, where $L_{\mathrm{m}}^{-1}$ is the inverse of the monaural loudness function. The decibel difference, then, between $P_{\mathrm{x}}$ and $P_{\mathrm{s}}$ is $20 \log \left\{L_{\mathrm{m}}^{-1}\left[2 L_{\mathrm{m}}\left(P_{\mathrm{s}}\right)\right] / P_{\mathrm{s}}\right\}$. Now, in the special case where $L_{\mathrm{m}}(P)$ is a power function of sound pressure, it is easy to show that the decibel difference should be $20 \log (2) / n$, where $n$ is the exponent of the power function. For $1-\mathrm{kHz}$ pure tones, the exponent of the loudness function obtained from magnitude-estimation experiments is around 0.6 (Marks, 1979a). Therefore, for pure tones, we would expect an approximately 10-dB difference between the binaural tone and the monaural tone that matched it in loudness. Note, however, that this 
expectation is based on three assumptions: ear symmetry, binaural additivity, and a monaural power function for loudness.

A number of studies have indicated that over a reasonable range of intensities (40-90 dB), there is an approximately $10-\mathrm{dB}$ difference between a binaural tone and its matched monaural tone (Marks, 1978,1980). For broadband noises, however, the separation between binaural and matched monaural noise increases from about $3 \mathrm{~dB}$ at $30 \mathrm{~dB}$ SPL to as much as $14 \mathrm{~dB}$ at $90 \mathrm{~dB}$ SPL (Marks, 1980; Scharf, 1968; Scharf \& Fishken, 1970). Clearly, for noises, one or more of the three assumptions upon which the prediction of a constant decibel difference is based is violated. With reference to our study, it is reasonably clear that the ears are not always symmetrical. Therefore, it is not required that the monaural-binaural difference be independent of intensity.

It is also true that loudness of a broadband noise deviates from a power function (Scharf \& Fishken, 1970; Zwicker \& Scharf, 1965). Although Figure 1 shows that a power function apparently describes the growth of loudness quite well, it is important to note that the present technique cannot distinguish between a loudness function of the form $L(P)=k P^{n}$ and one in which $L(P)=k P^{n}+b$, because the projection values are only linearly related to loudness. If $b$ is not zero, then a plot of log loudness versus $\log$ intensity will not be linear. Indeed, several studies have indicated that the loudness of a broadband noise is nonlinearly related to intensity in $\log -\log$ coordinates, a finding in accord with many other psychophysical studies as well as physiological data (e.g., Scharf \& Fishken, 1970; Zwicker \& Scharf, 1965). Figure 2 presents some data that are typical of the type of relation often observed in a magnitude-estimation experiment (from Marks, 1980, Experiments 1 and 2). The smooth curve fit to the data points is a function of the form $L(P)=k P^{n}+b$, where $n=.35, k=.44$, and $b$ is -1.11 . Clearly, a power function with an additive constant describes the data quite well. If we now use this function for loudness to predict the monaural match to a binaural noise, we no longer predict a constant difference as a function of intensity but a decibel difference that increases from about $5 \mathrm{~dB}$ at $30 \mathrm{~dB}$ to $16 \mathrm{~dB}$ at $90 \mathrm{~dB}$. This difference between monaural and matched binaural intensities is indeed very close to that found by Marks in his experiment. Therefore, the present experiment, which supports binaural additivity for noises, is consistent with the data from loudness matching either if we assume that monaural loudness of noises is not a simple power function of loudness but one that is approximated by the function $k P^{n}+b$ or if we assume that the two ears do not contribute equally to binaural loudness.

Indeed, a reexamination of the data of Levelt et al. (1972) indicates that, for both of their subjects, right-ear loudness contributed more to binaural loudness than leftear loudness for in-phase tones. Marks $(1978,1980)$ did not present scale values for left and right ears separately, so it was not possible to determine whether one ear contributed more to loudness than the other ear. Thus, the only

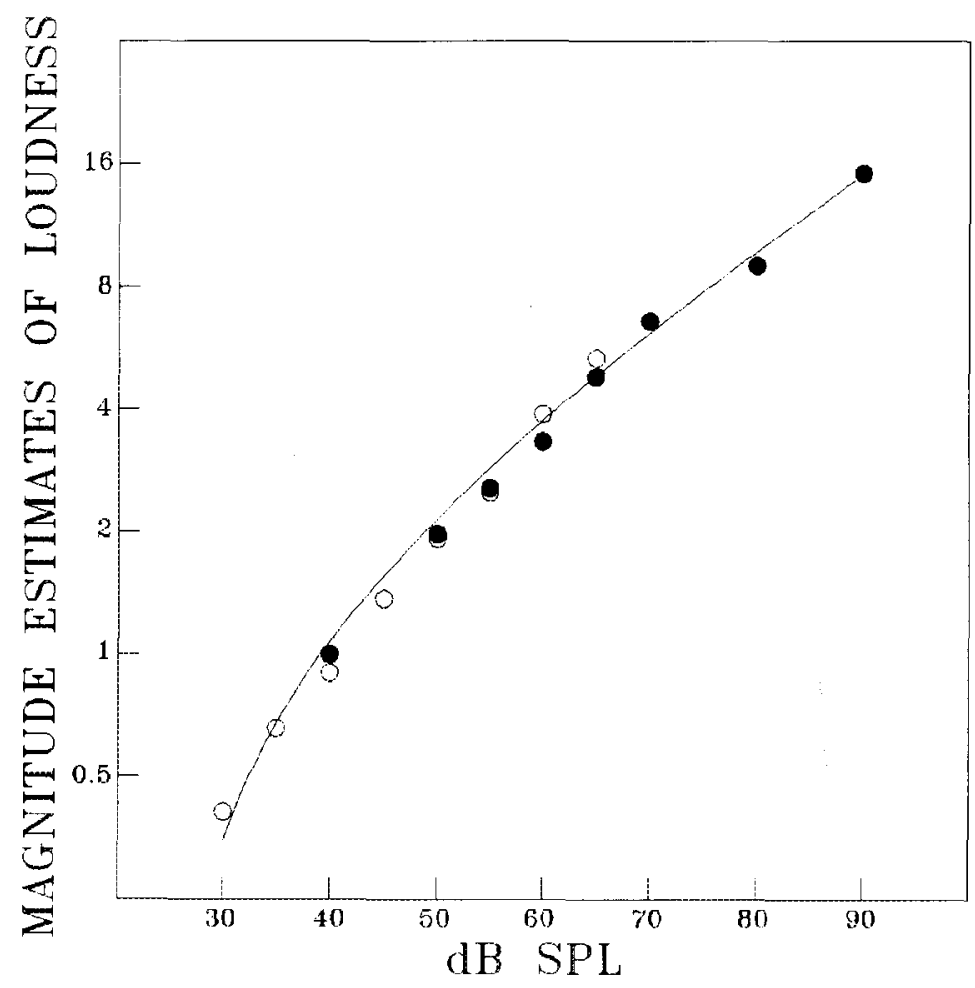

Figure 2. Magnitude estimates of the loudness of a monaural noise obtained in two experiments. Data are from Marks (1980) and were taken from his Figure 3. The smooth curve fit to the function is of the form $M E=k P^{n}+b$. 
previous instance in which right- and left-ear contributions to binaural loudness were measured in a conjoint paradigm (Levelt et al., 1972) also indicated that when the sounds were in phase, the right ear tended to contribute more to binaural loudness than did the left ear.

\section{Developmental Changes}

The rate of failure for transitivity and double cancellation for children is nearly as low as that for adults and provides strong support for an additive model for binaural loudness in children. Given that the paired comparison results for children also satisfy the conditions for an additive model, we can consider the projection values obtained from these data as representing interval scale measurement of loudness. Note that in constructing these interval scales of loudness for children we required a simple judgment on their part (which of two sounds was louder). That children were able to comply with these instructions is indicated by the fact that they averaged $92.6 \%$ on control trials (trials that presented pairs of sounds for which the direction of the loudness judgment was known a priori). Morover, a comparison of pairs in which the left- or right-ear intensity of one member of the pair was at least $12 \mathrm{~dB}$ greater than either the left- or right-ear intensity of the other member of the pair indicated that children could make consistent judgments of loudness even when the sounds to be compared differed in laterality. In such pairs, we would expect the member with the greater left- or right-ear intensity to be judged the louder. The percentage of times that children judged the member with the greater left- or right-ear intensity as louder was nearly as large for children $(90.4 \%$ and $90.0 \%$ for in-phase and counterphase noise, respectively) as for adults $(96.6 \%$ and $96.7 \%$, respectively). The success of this technique, then, is due to the use of a judgment that is well within the cognitive capacity of the children.

The loudness functions obtained for both left and right ears in children for in-phase noise were virtually identical. For the adults, the right-ear loudness function has a slightly greater range and steeper exponent than the leftear function. This suggests that, relative to children, adults favor the right ear in computing the binaural loudness of in-phase noises. To check whether this was indeed true, we examined those comparisons among in-phase noises where adults and children disagreed. Of the 11 comparisons in which they disagreed, adults chose the pair with the higher right-ear intensity exactly 8 times, which was not significant according to a binomial test $(p>.05$, twotailed). Thus, although there is an indication that adults favor the right ear more than do children for in-phase noise, this trend is not significant.

The loudness functions obtained for both left and right ears in adults for counterphase noise were virtually identical. For children, however, the left-ear loudness function had a greater range and steeper exponent than the rightear loudness function. Again, this suggests that adults, relative to children, give slightly more weight to the right ear than to the left ear in computing the binaural loudness of counterphase noises. When we examined the $15 \mathrm{com}-$ parisons on which adults and children disagreed, we found that adults chose the pair with the higher right-ear intensity in exactly 11 of those 15 times. By a binomial test, this again was not significant ( $p>.1$, two-tailed). If, however, we group the disagreements from both the in-phase and counterphase conditions, then the propensity for adults to favor the right ear relative to the performance of children is statistically significant ( 19 out of $26, p<$ .05 , two-tailed). Thus, there is some indication that as individuals age they tend to favor the right ear slightly more with respect to binaural loudness.

\section{Ear Dominance and Phase}

Both children and adults apparently favor the right ear more in computing binaural loudness when the noises are in phase than when they are in counterphase. However, it is possible that the right-ear advantage for in-phase noise is an artifact resulting from comparing the loudnesses of sounds that are lateralized on different sides of the head. As noted earlier, in all of the experimental comparisons, the two in-phase sounds to be compared will be lateralized to different sides of the head. Suppose there is a bias to judge a sound experienced on the right side of the head as louder than an equivalent sound on the left side of the head. ${ }^{1}$ This would result in a tendency to judge the sound having the higher right-ear intensity as louder for in-phase conditions. However, when the sounds are in counterphase, they will be experienced as more diffuse and will be less precisely lateralized. Therefore, for counterphase sounds, we will expect less precise lateralization and a diminution of the hypothesized right-ear bias. To check on whether or not listeners have a side bias, we conducted the following control experiment.

The control experiment employed six left-ear and six right-ear noises with the same spectral characteristics and sound pressure levels as those used in Experiments 1 and 2 . Twenty adult listeners were presented with comparisons between one of the six left-ear noises and one of the six right-ear noises in a two-interval forced choice paradigm (one sound in each interval) and asked to indicate which of the two sounds was louder. Note that, because the sounds were monaural and presented sequentially, these loudness comparisons are between one sound lateralized to the right and another lateralized to the left. Each listener was presented with each of the 36 possible pairs of the left- and right-ear noises (randomly ordered) a total of six times in a single session. After completing the first session, they were also tested in another session. When the left-ear signal was more intense, subjects identified the left-ear signal as louder $86.8 \%$ of the time. When the right-ear signal was more intense, they identified the right-ear signal as louder $85.9 \%$ of the time. Thus, there was no evidence of an ear bias when the sounds were unequal. For the six pairs of the sounds whose right- and leftear intensities were identical, listeners judged the rightear sound to be more intense than the left-ear sound on $51 \%$ of the trials in the first session and on $48 \%$ of the trials in the second session. Of the 20 listeners, 9 favored the right-ear sound more than $50 \%$ of the time, $10 \mathrm{fa}$ - 
vored the left-ear sound more than $50 \%$ of the time, and 1 judged the right-ear sound as louder than the left-ear sound exactly $50 \%$ of the time. Thus, there was no indication that sounds on the right side are judged as louder than sounds on the left side.

It appears, then, that for broadband noise, reversing the phase affects the relative contribution of the two ears to binaural loudness. The latter result was totally unexpected. It has long been known that the right ear (left hemisphere) is dominant for dichotic presentations of speech (see Bryden, 1982, for a review), and there are even some indications of left-ear dominance for some, but not all, nonspeech sounds such as musical chords (Bryden, 1982, pp. 39-66). However, we are not aware of any accounts that suggest that a change of phase can produce a change in ear dominance.

In most previous accounts of ear dominance, it is assumed that the reason for the dominance was related to the cortical area responsible for processing the stimuli in question. Thus, if the stimuli were speech or speech-like, it was assumed that the right ear dominated in dichotic listening tasks because speech is processed by the left hemisphere. It is unlikely that a similar explanation can be applied to the present results. First, the stimuli do not resemble speech material. Second, there is no reason to believe that the hemisphere primarily responsible for the processing of the sounds changes with a change in the interaural phase of the sound. For these reasons, it is unlikely that this phase effect is related to hemispheric specialization. What is more likely is that the right-ear bias for in-phase noises is related to the effect that interaural phase has on the degree of binaural summation (Mulligan, Goodman, Gleisner, \& Faupel, 1985). The precise nature of the relation, however, remains to be determined.

\section{REFERENCES}

Algom, D., \& MARKs, L. E. (1984). Individual differences in loudness processing and loudness scales. Journal of Experimental Psychology: General, 113, 571-593.

ANDERSON, N. H. (1970). Functional measurement and psychophysical judgment. Psychological Review, 77, 153-170.

ANDERSON, N. H. (1974). Algebraic models in perception. In E. C. Carterette \& M. P. Friedman (Eds.), Handbook of perception: Vol. II. Psychophysical judgment and measurement (pp. 215-298). New York: Academic Press.

Bissetr, R., \& Schneider, B. (1991). Spatial and conjoint models based on pairwise comparisons of dissimilarities and combined effects: Complete and incomplete designs. Psychometrika, 56, 685-698.

Bond, B., \& STEvens, S. S. (1969). Cross-modality matching of brightness to loudness by 5-year-olds. Perception \& Psychophysics, 6 , 337-339.

BRYDEN, M. P. (1982). Laterality: Functional asymmetry in the intact brain. New York: Academic Press.

Carterette, E. C., \& Anderson, N. H. (1979). Bisection of loudness. Perception \& Psychophysics, 26, 265-280.

Collins, A. A., \& Gescheider, G. A. (1989). The measurement of loudness in individual children and adults by absolute magnitude estimation and cross-modality matching. Journal of the Acoustical Society of America, 85, 2012-2021.

Curtis, D. W., AtTNeave, F., \& Harrington, T. L. (1968). A test of a two-stage model of magnitude judgment. Perception \& Psychophysics, 3, 25-31.

Curtis, D. W., \& Rule, S. J. (1972). Magnitude judgments of lightness and brightness difference as a function of background reflectance. Journal of Experimental Psychology, 95, 215-222.

Durlach, N. I., \& Colburn, H. S. (1978). Binaural phenomena. In E. C. Carterette \& M. P. Friedman (Eds.), Handbook of perception. Vol. IV. Hearing (pp. 365-466). New York: Academic Press.

FaGot, R. F., SteWART, M. R., \& Kleinknecht, R. E. (1975). Representations for biased numerical judgments. Perception \& Psychophysics, 17, 309-319.

Gigerenzer, G., \& Strube, G. (1983). Are there limits to binaural additivity of loudness? Journal of Experimental Psychology: Human Perception \& Performance, 9, 126-136.

IRWIN, R. J. (1965). Binaural summation of thermal noises of equal and unequal power in each ear. American Journal of Psychology, 78, 57-65.

JENSEN, K. J., \& NEFF, D. L. (1993). Development of basic auditory discrimination in preschool children. Psychological Science, 4, 104-107.

Krantz, D. H., Luce, R. D., Suppes, P., \& TVersky, A. (1971). Foundations of measurement: Vol. I. Additive and polynomial representations. New York: Academic Press.

Levelt, W. J. M., Riemersma, J. B., \& Bunt, A. A. (1972). Binaural additivity of loudness. British Journal of Mathematical \& Statistical Psychology, 25, 51-68.

LUCE, R. D., \& TUKEY, J. W. (1964). Simultaneous conjoint measurement: A new type of fundamental measurement. Journal of Mathematical Psychology, 1, 1-27.

MARKS, L. E. (1978). Binaural summation of the loudness of pure tones. Journal of the Acoustical Society of America, 64, 107-113.

Marks, L. E. (1979a). Sensory and cognitive factors in judgments of loudness. Journal of Experimental Psychology: Human Perception \& Performance, 5, 426-443.

MARKs, L. E. (1979b). A theory of loudness and loudness judgments. Psychological Review, 86, 256-285.

MARKS, L. E. (1980). Binaural summation of loudness: Noise and twotone complexes. Perception \& Psychophysics, 27, 489-498.

MAXON, A. B., \& HochBerg, I. (1982). Development of psychoacoustic behavior: Sensitivity and discrimination. Ear \& Hearing, 3, 301-308.

Mulligan, B. E., Goodman, L. S., Gleisner, D. P., \& Faupel, M. L. (1985). Steps in loudness summation. Journal of the Acoustical Society of America, 77, 1141-1154.

Rule, S. J., Curtis, D. W., \& Markley, R. P. (1970). Input and output transformations from magnitude estimation. Journal of Experimental Psychology, 86, 343-349.

Rule, S. J., LAYE, R. C., \& CurTis, D. W. (1974). Magnitude judgments and difference judgments of lightness and darkness: A two-stage analysis. Journal of Experimental Psychology, 103, $1108-1114$.

SCHARF, B. (1968). Binaural loudness summation as a function of bandwidth. In Y. Kohasi (Ed.), Reports of the 6th International Congress on Acoustics (Vol. 1, pp. 25-28). (Paper A-35)

Scharf, B., \& FishKeN, D. (1970). Binaural summation of loudness: Reconsidered. Journal of Experimental Psychology, 86, 374-379.

SCHNEIDER, B. (1988). The additivity of loudness across critical bands: A conjoint measurement approach. Perception \& Psychophysics, 43, 211-222.

Schneider, B., Parker, S., Valenti, M., Farrell, G., \& Kanow, G. (1978). Response bias in category and magnitude estimation of difference and similarity for loudness and pitch. Journal of Experimental Psychology: Human Perception \& Performance, 4, 483-496.

STEVENS, S. S. (1956). The direct estimation of sensory magnitudeloudness. American Journal of Psychology, 69, 1-25.

TEGHTSOONIAN, M. (1980). Children's scales of length and loudness: A developmental application of cross-modal matching. Journal of Experimental Child Psychology, 30, 290-307.

ZWICKER, E., \& SCHARF, B. (1965). A model of loudness summation. Psychological Review, 72, 3-26.

\section{NOTE}

1. We would like to thank Bertram Scharf for pointing out this possibility to us.

(Manuscript received September 30, 1994; revision accepted for publication August 6, 1996.) 